\title{
Symmetric surface waves along a metamaterial dielectric waveguide and a perfectly conducting cylinder covered by a metamaterial layer
}

\author{
Y. Shestopalov ${ }^{1}$ and E. Kuzmina ${ }^{2}$ \\ ${ }^{1}$ University of Gävle, Sweden \\ ${ }^{2}$ Moscow Technological University MIREA, Russia \\ E-mail: yuyshv@hig.se, ekaterina.kuzm@gmail.com
}

\begin{abstract}
Existence of symmetric complex waves in a metamaterial dielectric rod and a perfectly conducting cylinder of circular cross section covered by a concentric layer of metamaterial, a metamaterial Goubau line, is proved. Analytical investigation and numerical solution of dispersion equations reveal several important properties of running waves inherent to open metal-metamaterial waveguides which have not been reported for waveguides filled with standard media.
\end{abstract}

\section{Introduction}

In this study we develop a mathematical model describing the propagation of radially symmetric surface waves along a metamaterial dielectric rod (DR) and a perfectly conducting cylinder of circular cross section covered by a concentric layer of metamaterial, the Goubau line (GL).

The metamaterial is characterized by negative relative permittivity and permeability and simulates artificial media of different types [1]. Interaction between electromagnetic field and metamaterial is expected to produce and is associated with various phenomena, e.g. negative refraction [2]. In this work, we discover several such phenomena which have not been reported for waveguides filled with standard media having positive permittivity.

Metamaterials are lossy dispersive media characterized by generally complex permittivity and permeability varying w.r.t. frequency, so that permeability and the real and imaginary parts of permittivity take negative and positive values and vanish at certain frequency points [1]-[3]. However, at certain frequency intervals these dependence may be virtually constant [3] so that one may ignore the dispersion of a metamaterial. In this work, we study such cases of constant negative permittivity and permeability in a prescribed frequency range. This is the first and necessary step to proceed to the analysis of dispersive lossy metamaterial open waveguides.

Investigation of the wave propagation in open metalmetamaterial waveguides requires elaboration of specific mathematical and numerical methods based on (i) proper reformulation of the corresponding boundary eigenvalue problems equipped with appropriate conditions at infinity that enable one to take into account all possible types of real (and complex) waves, (ii) investigation of the boundary eigenvalue problem spectra, and (iii) analysis of dis- persion equations (DEs) using the theory of functions of several complex variables and perturbation techniques. In the case of a metamaterial cover, it is necessary to reconsider properties of the functions entering DEs so that the $\mathrm{DE}$ analysis becomes a central point of the present work. This analysis is performed using the results obtained earlier in [4] and [5] for the real and complex symmetric and nonsymmetric waves in 'standard' GL and DR. Numerical algorithms and codes are created for calculating propagation constants and fields in the considered metalmetamaterial waveguide and the results of sample computations and numerical modeling are presented.

It should be noted that rigorous mathematical proof of the existence of real and complex waves in metamaterial DR and GL remains, to the best of our knowledge, one of unsolved problems of the theory of electromagnetic wave propagation in open waveguides. These gaps in the theory have driven us to elaborate in this work appropriate mathematical methods and complete the proofs beginning from basic open metal-dielectric structures, DR and GL.

Give a short outline of this work: in Section 1 we formulate the problems under study. In Section 2 we summarize the results for symmetric surface waves in DR and GL filled with 'standard' dielectrics. In Sections 3 and 4 we prove the existence and investigate basic properties of symmetric surface waves in metamaterial DR and GL using the methods [4], [5] and [8] by investigating roots of the DEs that describe symmetric waves w.r.t. all the problem parameters.

\section{Statement}

Consider the propagation of symmetric waves described in terms of nontrivial solutions to homogeneous Maxwell's equations in DR and GL depicted in Fig. 1 where all the geometric parameters are defined. The media filling DR and GL situated in vacuum are assumed to be metamaterials having negative constitutive parameters (permittivity and permeability) $\epsilon<0$ and $\mu<0$, so that the optical quantity, the medium refractive index $n=-\sqrt{\epsilon \mu}<0$ and $n^{2}=\epsilon \mu>0$ and longitudial wavenumber $k_{s}$ of the symmetric waves

$$
k_{s}^{2}=\left\{\begin{array}{l}
k_{0}^{2}-\beta^{2}, \quad r>a(\mathrm{DR}) \text { or } r>b(\mathrm{GL}), \\
k_{0}^{2} n^{2}-\beta^{2}, \quad r<a(\mathrm{DR}) \text { or } a<r<b(\mathrm{GL}),
\end{array}\right.
$$



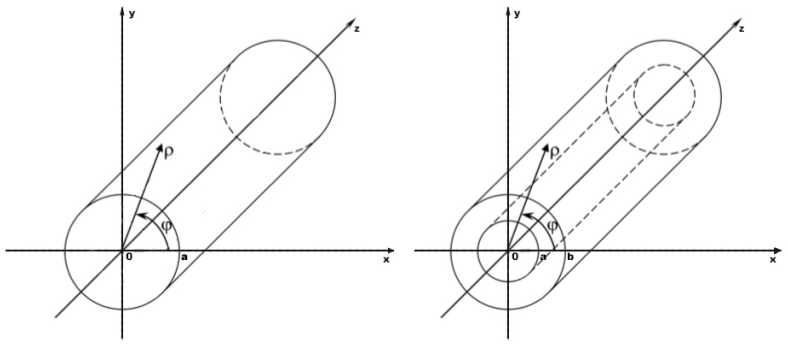

Figure 1: Dielectric rod and Goubau line.

Here, $\beta$ is the wave propagation constant (spectral parameter), and $k_{0}$ is the free-space wavenumber. The symmetric (azimuthally-independent) waves have the nonzero components

$$
\begin{gathered}
\mathbf{H}=\left[0, H_{2}(r, z), 0\right], \mathbf{E}=\left[E_{1}(r, z), 0, E_{3}(r, z)\right], \\
E_{1}=-\frac{i \beta}{k_{s}^{2}} \frac{d \phi}{d r} e^{-i \beta z}, E_{3}=\phi(r) e^{-i \beta z}, \\
H_{2}=-\frac{i \omega \epsilon}{k_{s}^{2}} \frac{d \phi}{d r} e^{-i \beta z},
\end{gathered}
$$

where $\phi$ solves boundary eigenvalue problems

$$
\begin{aligned}
& \mathcal{L} \phi \equiv \frac{1}{r} \frac{d}{d r}\left(r \frac{d \phi}{d r}\right)+k_{s}^{2} \phi=0, \quad r>0, \\
& {\left.[\phi]\right|_{r=a}=\left.\left[\frac{\epsilon}{k_{s}^{2}} \frac{d \phi}{d r}\right]\right|_{r=a}=0, }
\end{aligned}
$$

for DR and

$$
\begin{aligned}
\mathcal{L} \phi=0, \quad r>a, & \phi(a)=0,\left.[\phi]\right|_{r=b}= \\
& =\left.\left[\frac{\epsilon}{k_{s}^{2}} \frac{d \phi}{d r}\right]\right|_{r=b}=0
\end{aligned}
$$

for GL. Problems (3) and (4) must be complemented with the conditions at infinity: the condition

$$
\phi(r) \rightarrow 0, \quad r \rightarrow \infty
$$

gives rise to symmetric surface waves in DR and GL described in terms of real-valued quantities; in particular, the boundary operators in (3) are defined (as real-valued functions of a real variable $\gamma$ or $\lambda=\gamma^{2}$ ) on a certain interval $I$ specified below. In [5] it is proved that the spectrum of surface waves in DR and GL filled with homogeneous dielectric may be empty, or they may consist of several (real) points located on this interval.

Make the following designations:

$\gamma=\frac{\beta}{k_{0}}, \kappa=k_{0} a, x=\kappa \sqrt{n^{2}-\gamma^{2}}, u=\kappa \sqrt{n^{2}-1}$,

$w=\sqrt{u^{2}-x^{2}}=\kappa \sqrt{\gamma^{2}-1}$.
For surface waves, potential function $\phi(r)$ can be represented, according to the conditions at infinity (5), as

$$
\begin{aligned}
& \phi(r)=\left\{\begin{array}{l}
A K_{0}\left(\frac{r}{a} w\right), \quad r>a, \quad(\mathrm{DR}), \\
B J_{0}\left(\frac{r}{a} x\right), \quad r<a,
\end{array}\right. \\
& \phi(r)=\left\{\begin{array}{l}
A K_{0}\left(\frac{r}{a} w\right), \quad r>b, \\
\frac{B}{Y_{0}(x)}\left[J_{0}\left(\frac{r}{a} x\right) Y_{0}(x)-Y_{0}\left(\frac{r}{a} x\right) J_{0}(x)\right], \\
a<r<b(\mathrm{GL}) .
\end{array}\right.
\end{aligned}
$$

Here, $J_{k}(x), Y_{k}(x), K_{0}(x)$, and $K_{1}(x)$ denote, respectively, the Bessel and Neumann functions of the order $k=$ 0,1 and the McDonald functions. The form of the solutions in unbounded regions is governed by asymptotic representation at large $|z|$ of McDonald function $K_{0}(z)$.

To investigate complex symmetric waves complement (3) with a condition at infinity [7]

$$
\begin{aligned}
\phi(r) & =A H_{0}^{(1)}(r \tilde{w}), r>a(\mathrm{DR}) \text { or } r>b(\mathrm{GL}), \\
\tilde{w} & =\frac{i}{a} \sqrt{u^{2}-x^{2}}=k_{0} \sqrt{1-\gamma^{2}} .
\end{aligned}
$$

Here $\gamma$ may be complex quantity and $\tilde{w}$ is the transverse wavenumber of the medium outside DR (vacuum).

In view of the logarithmic singularity of the Hankel function $H_{0}^{(1)}(z)$ at $z=0$, eigenvalue problem (3), (7) and (4), (7) are considered, following [7] at $\gamma \in \Lambda$, where $\Lambda$ is the multi-sheet Riemann surface of the function $f(\gamma)=$ $\ln \sqrt{1-\gamma^{2}}$.

Denote by $\Lambda_{0}$ the principal ('proper') sheet of this Riemann surface specified by the condition $\Im w \geq 0$.

List the properties [9] of symmetric wave spectrum in a DR filled with homogeneous dielectric medium having constant permittivity $\epsilon$ : (i) The imaginary and real axes of the sheet $\Lambda_{0}$ except the interval $\gamma \in I_{0}=(1, \sqrt{\epsilon})$ do not contain eigenvalues of problem (3), (7). (ii) Interval $I_{0}$ contains a finite number (or none) of eigenvalues; their number depends on the problem parameters and is clarified in the next sections. (iii) Surface and complex waves of DR correspond to real eigenvalues $\gamma \in I_{0}$ and complex eigenvalues $\gamma \in \Lambda_{0}$, respectively. Leaky eigenmodes correspond to complex eigenvalues $\gamma$ belonging to an improper sheet of $\Lambda$ for which $\Im w<0$.

\section{Symmetric surface waves in DR and GL with positive permittivity}

By applying to (6) the conditions of continuity of $\phi$ we obtain the DEs: for DR

$$
G_{d}(x, u)=F_{d}(x),
$$

or

$$
F_{D}(x, u)=0, \quad F_{D}(x, u) \equiv G_{d}(x, u)-F_{d}(x),
$$

where

$$
\begin{aligned}
G_{d}(x, u) & =-\epsilon w \frac{K_{0}(w)}{K_{1}(w)} \\
F_{d}(x) & =x \operatorname{cotj}(x), \cot (x):=\frac{J_{0}(x)}{J_{1}(x)}
\end{aligned}
$$


for GL,

$$
F_{G}(x, u, s) \equiv G_{g}(x, u, s)-F_{g}(x, s)=0 .
$$

where

$$
\begin{gathered}
G_{g}(x, u, s)=\epsilon \sqrt{u^{2}-x^{2}} \frac{K_{0}\left(s \sqrt{u^{2}-x^{2}}\right)}{K_{1}\left(s \sqrt{u^{2}-x^{2}}\right)}, \\
F_{g}(x, s)=x \operatorname{Cotj}(x), \quad \operatorname{Cotj}(x)=\frac{\Phi_{1}(x, s)}{\Phi_{2}(x, s)}, \\
\Phi_{1}(x, s)=J_{0}(s x) Y_{0}(x)-J_{0}(x) Y_{0}(s x), \\
\Phi_{2}(x, s)=J_{0}(x) Y_{1}(s x)-J_{1}(s x) Y_{0}(x) .
\end{gathered}
$$

The existence of real symmetric surface waves in DR and GL filled with homogeneous dielectric medium having positive constant permittivity is proved [5] by considering equations (9) and (11) w.r.t. the (real) variable

$$
x=\kappa \sqrt{(\epsilon-1)-w^{2}}=\kappa \sqrt{\epsilon-\gamma^{2}}, \quad x \in(0, \kappa \sqrt{\epsilon-1})
$$

where $\epsilon \geq 1$. If $x^{*} \in(0, \kappa \sqrt{\epsilon-1})$ is a root of (9), then

$$
\gamma^{*}=\sqrt{\epsilon-\left(\frac{x^{*}}{\kappa}\right)^{2}} \in(1, \sqrt{\epsilon})
$$

is the propagation constants of a real symmetric surface wave. The analysis in [5] is largely based on detailed investigation of cylindrical cotangent functions $\operatorname{cotj}(x)$ and $\operatorname{Cotj}(x)$ that exhibit all basic properties of the trigonometric cotangent; namely, $\operatorname{cotj}(x)$ takes all real values on every interval $\left(\nu_{m}^{1}, \nu_{m+1}^{1}\right), \operatorname{cotj}(x)>0, x \in\left(\nu_{m}^{1}, \nu_{m}^{0}\right)$, and $\operatorname{cotj}\left(\nu_{m}^{n}\right)=0$, where $\nu_{m}^{n}, m=0,1,2, \ldots$, are the $m$ th zeros of Bessel functions $J_{n}(x)(n=0,1)$. The same applies to $\operatorname{Cotj}(x)$ on intervals $\left(h_{n}^{1}(s), h_{n+1}^{1}(s)\right)$ and $\left(h_{n}^{1}(s), h_{n}^{0}(s)\right)$ where $h_{n}^{0}(s)$ and $h_{n}^{1}(s)$ are the $n$th zeros of, respectively, $\Phi_{2}(x, s)$ and $\Phi_{1}(x, s)$ w.r.t. $x$ with $s>0$.

According to [5], if $u=\kappa \sqrt{\epsilon-1} \geq \nu_{1}^{0}$, then there exists a root $x=x^{*} \in\left(\nu_{1}^{0}, \nu_{1}^{1}\right)$ of $\mathrm{DE}(9)$; if $u \in\left(\nu_{1}^{0}, \nu_{1}^{1}\right)$, then (9) has only one root. Thus there are at most finitely many symmetric surface waves propagating in a lossless ( $\varsigma \epsilon=0, \Re \epsilon>1$ ) DR and their normalized propagation constants $\gamma \in \Gamma(\epsilon)=(1, \sqrt{\epsilon})$. The symmetric surface waves in DR are absent if $u<\nu_{1}^{0}$, so that for every $\epsilon>1$ there is a $\kappa=\kappa_{*}(\epsilon)>0$ such that DE (9) has no roots on the interval $\Gamma(\epsilon)$ for $\kappa \in\left(0, \kappa_{*}(\epsilon)\right)$.

For GL, there exists a root $x^{1}=x^{1}(s)$ of DE (11) at an arbitrary $s=b / a>1$, so that GL supports (at least one) symmetric fundamental surface wave (the so-called Sommerfeld wave) for arbitrarily thin dielectric cover and any $\epsilon>1$. Higher-order surface waves exist under the condition

$$
u=\kappa \sqrt{\epsilon-1} \geq h_{1}^{0}(s) .
$$

with $x \in(0, \kappa \sqrt{\epsilon})$, in particular, $N$ symmetric surface waves for $u \geq h_{N}^{0}(s)(N=1,2, \ldots)$.

\section{Symmetric surface waves in metamaterial DR and GL}

Having elaborated the analytical and numerical methods for determining real and complex waves in homogeneously filled DR and GL, we extend the technique and consider these waveguides filled with metamaterial media. To this end, one can consider DEs (9) and (11) w.r.t. the real variable $x=\kappa \sqrt{n^{2}-\gamma^{2}}$. The proof of existence and determination of the intervals of localization of real roots of DEs (9) and (11) can be performed similar to [5]: by analyzing the behavior of the right- and left-hand sides of (9) and (11) w.r.t. $x \in(1, n)$ for a given constant $n>1$; the only difference is that in (9) and (11), parameter $\epsilon<0$.

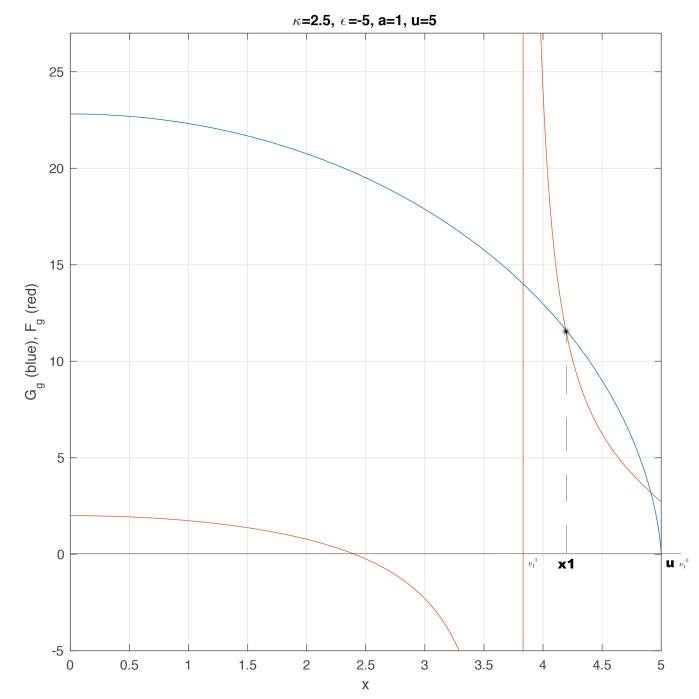

Figure 2: Graphs show the location of the first two roots of $\mathrm{DE}$ (9) w.r.t. $x$ for $\kappa=2.5, \epsilon=-5, a=1, u=5$.

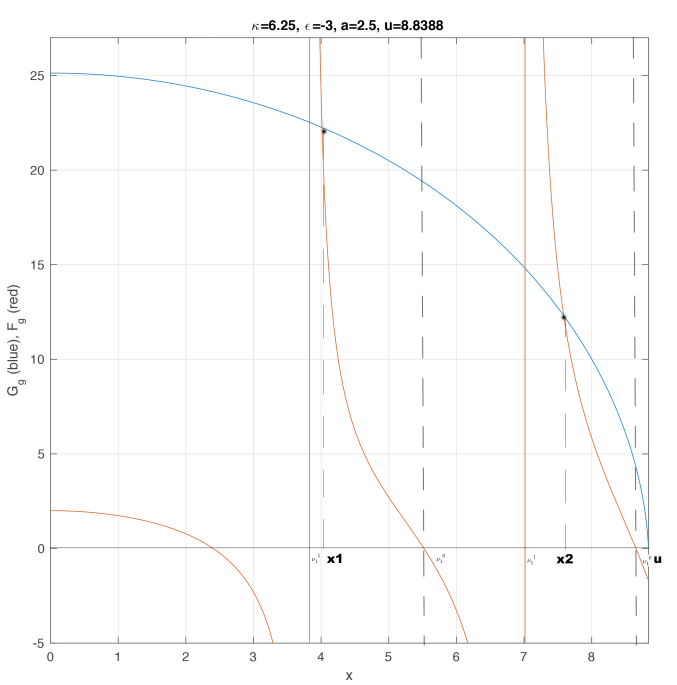

Figure 3: Graphs show the location of the first two roots of DE (9) w.r.t. $x$ corresponding to higher-order symmetric surface waves for $\kappa=6.25, \epsilon=-3, a=2.5, u=8.8388$. 
Numerical solution of DEs (9) and (11) is performed at constant negative values of permittivity and permeability, disregarding thus frequency dispersion of the metamaterial. For sample calculations, we choose DR and GL and a range of values for their metamaterial core and cover $\epsilon=-5$ and $\mu=-1$ resulting in $n^{2}=5$ that occurs at the frequencies $f=f^{*} \sim 12 G H z$ obtained for a double-ring-resonator metamaterial described in [3]. Other parameter values are $k_{0}=k_{0}^{*}=\frac{2 \pi f^{*}}{c} \approx \frac{2 \pi 12 \cdot 10^{9}}{3 \cdot 10^{10}}=0.8 \pi \approx 2.5$ yielding $\kappa^{*}=k_{0}^{*} a=1$ at the DR radius $a=\frac{1}{k_{0}^{*}}=0.4[\mathrm{~cm}]$.

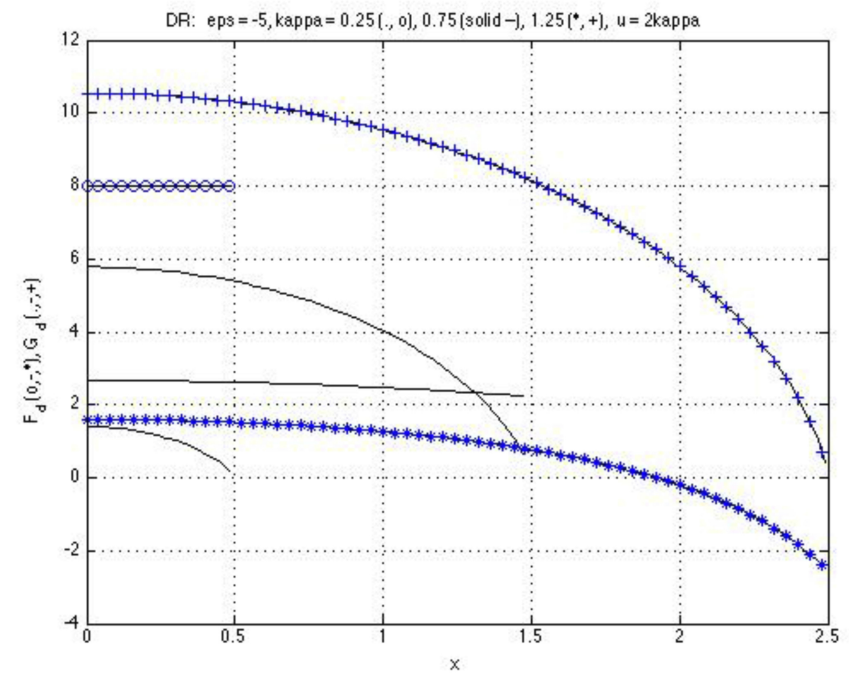

Figure 4: Graphs of $G_{d}(x, u)(.,-,+)$ and $F_{d}(x)\left(o,-,{ }^{*}\right)$ against $x$ for $\epsilon=-5, \kappa=0.25$ (., o, curves on the smallest interval $x \in(0, u), u=2 \kappa=0.5), 0.75(-)$, and $1.25\left(^{*}\right.$, + , curves on the largest interval $x \in(0, u), u=2 \kappa=2.5)$ illustrating the absence and the evolution (removal) of the root of DE (8) for the fundamental DR surface mode as $\kappa$ increases.

\subsection{Metamaterial DR}

To determine the conditions of the existence of symmetric surface waves in DR for negative $\epsilon$ resulting from the occurrence of roots of DE (9) on the interval $\Gamma(\epsilon)$ where DE is defined and considered, highlight some properties of the functions $F_{d}=F_{d}(\gamma)$ and $G_{d}=G_{d}(\gamma)$ entering $\operatorname{DE}(9)$ :

(id) $G_{d}(\gamma)$ is bounded and continuous on $\Gamma(\epsilon), G_{d}(\gamma=$ $1)=0, G_{d}(\gamma)$ decreases monotonically and is negative on $\Gamma(\epsilon)$ for all $\kappa>0, G_{d}(\kappa=0)=0$ for all $\gamma \in \Gamma(\epsilon)$, and the range of $G_{d}(\gamma)$ is the interval $\mathcal{K}(\epsilon, \kappa)=\tilde{\mathcal{K}}(u)=$ $\left\{y=G_{d}(\gamma): 0<y<\mathrm{K}(u)\right\}$ where $\mathrm{K}(u)=\epsilon u \frac{K_{0}(u)}{K_{1}(u)}<$ 0 and $-\mathrm{K}(u)$ increases monotonically (for $\epsilon<-1$ and $u=\kappa \sqrt{|\epsilon|-1}>0$ ), and $\lim _{u \rightarrow+0} \mathrm{~K}(u)=0$ (in view of the asymptotic expansions of the modified Bessel functions in a vicinitiy of zero).

(iid) $-F_{d}(\gamma)$ is negative on $\Gamma(\epsilon)$ for sufficiently small $\kappa$ with $-F_{d}(\gamma=1)=\mathrm{F}(u)=-u \operatorname{cotj}(u)<0$ for $\kappa$ such that $0<u<\nu_{1}^{0} ; \lim _{x \rightarrow+0} x \operatorname{cotj}(x)=\lim _{x \rightarrow 0} x \frac{J_{0}(x)}{J_{1}(x)}=2$ (in view of the asymptotic expansions of the Bessel functions in a vicnitiy of zero), so that one can set $-F_{d}(\gamma=\sqrt{|\epsilon|})=-2$ for all $\kappa>0$ and $-F_{d}(\kappa=0)=-2$. Thus if $\kappa$ is small (tends to zero), the magnitude of $\min _{\Gamma(\epsilon)} G_{d}(\gamma)=\mathrm{K}(u)$ can be arbitraily small while $\left|F_{d}(\gamma)\right|$ is close to a constant 2 , so that $\mathrm{K}(u)>-2$ for $\kappa \in\left(0, \kappa_{*}\right)$ and $-F_{d}(\gamma)>G_{d}(\gamma)$ on the whole interval $\Gamma(\epsilon)$ for a certain $\kappa_{*}=\kappa_{*}(\epsilon)>0$.

(iiid) $F_{d}(\gamma)$ has singularities (poles) on $\Gamma(\epsilon)$ at $\gamma=$ $\gamma_{n, d}=\sqrt{\epsilon-\left(\frac{\nu_{n}^{1}}{\kappa}\right)^{2}}$ corresponding to $x=x_{n, d}=$ $\kappa \sqrt{\epsilon-\gamma_{n, d}^{2}}=\nu_{n}^{1}$, if $\kappa>0$ is such that $\gamma_{n, d} \in \Gamma(\epsilon)$ $(n=1,2, \ldots)$ for a given $\epsilon<-1$.

Thus, for every $\epsilon<-1$ there is a $\kappa=\kappa_{*}(\epsilon)>0$ such that DE (9) has no roots on the interval $\Gamma(\epsilon)$ for $\kappa \in\left(0, \kappa_{*}(\epsilon)\right)$. In Fig. 5 this case is illustrated by the curves plotting the functions $F_{d}=F_{d}(\gamma)$ and $G_{d}=G_{d}(\gamma)$ entering DE (9) at $\kappa=0.06$. When $\kappa$ increases (together with $u$ ), DE (9) acquires the root $\gamma_{0, d}^{(1)}=\sqrt{|\epsilon|-\left(\frac{x_{0, d}^{(1)}}{\kappa}\right)^{2}}$ with $0<x_{0, d}^{(1)}<\nu_{1}^{0}$ which has a lower cutoff $\kappa=\kappa_{*}(\epsilon)$; its value is approximately determined from the condition

$$
\mathrm{K}(u)=-2, \quad \mathrm{~K}(u)=\epsilon u \frac{K_{0}(u)}{K_{1}(u)}, \quad u=\kappa \sqrt{|\epsilon|-1},
$$

satisfied already at $\kappa=\kappa_{*}(-5) \approx 0.33$ and $\kappa=$ $\kappa_{*}(-10) \approx 0.13$.

This root is removed with a further increase of $\kappa$ (and $u$ ) so that it has an upper cutoff determined from the condition $u=\nu_{1}^{0}$ as can be followed by observing the mutual location of blue and red curves in Figs. 2 and 3. Figure 5 show plots of the functions $F_{d}=F_{d}(\gamma)$ and $G_{d}=G_{d}(\gamma)$ entering DE (9) on $\gamma \in \Gamma(\epsilon)$ for different values of $\kappa$. The plots enable one to follow emergence, evolution, and removal of the roots of DE (9) and thus emergence and removal of the fundamental (with no oscillations) DR surface mode. In particular, the upper red curve in Fig. 5 for $G_{d}(\gamma)$ and the curve marked with + for $F_{d}(\gamma)$ plotted for $\kappa=0.06$ do not intersect which indicates the absence of roots of DE (9) for small $\kappa \in(0,0.05)$.

Next, as $\kappa$ increases (as well as $u$ ), $|\mathrm{F}(u)|$ decreases approaching zero from below and DE (9) acquires two roots. $\gamma_{1, d}^{(q)}=\sqrt{|\epsilon|-\left(\frac{x_{1, d}^{(q)}}{\kappa}\right)^{2}}$ with $\nu_{1}^{1}<x_{1, d}^{(q)}<\nu_{2}^{0}, q=1,2$, where $x_{1, d}^{(1)}<x_{1, d}^{(2)}$ and $\gamma_{1, d}^{(1)}>\gamma_{1, d}^{(2)}$. The root $x_{1, d}^{(2)}$ is in a left vicinity of $x=u$ and the corresponding $\gamma_{1, d}^{(1)}$ is in a right vicinity of $\gamma=1$. $\gamma_{1, d}^{(2)}$ is displayed as a point of intersection of the middle solid curves in Figs. 4 and 5 and the second (right) intersection point of blue and red curves in Fig. 2. This intersection is preserved until $\mathrm{F}(u)$ vanishes at $u=\nu_{1}^{0}$ and then becomes positive (upper curves in Fig. 5. $|\mathrm{K}(u)|$ increases together with $\kappa$ which leads to the removal of the root of DE (9) indexed with $q=2$. While the root $\gamma_{1, d}^{(2)}$ (smaller w.r.t. $\gamma$ ) disappears as $\kappa$ increases, the second (greater w.r.t. $\gamma$ ) root $\gamma_{1, d}^{(1)}$ shifts towards the 
(first) singularity $\gamma_{1, d} \in \Gamma(\epsilon)$ of $F_{d}=F_{d}(\gamma)$ and remains for all $\kappa>0$. This root is clearly seen in Fig. 2 as the first (left) intersection point marked with a black dot resulting from a shift to the right of the blue $G_{d}(x)$-curve as $\kappa$ (and $u$ ) increase. The removal of the root $x_{1, d}^{(2)}$ which tends to $x=u$ (and $\gamma_{1, d}^{(2)}$ tends to $\gamma=1$ ) and then disappears as $\kappa$ increases can be easily traced in Figs. 3 and 4. Thus, for $\kappa \in\left(\kappa_{*}, \kappa_{*}^{1}\right)$ where $\kappa_{*}^{1}=\kappa_{*}^{1}(\epsilon)$ is such that $u=u_{*}^{1}=\kappa_{*}^{1}(\sqrt{|\epsilon|-1})=\nu_{1}^{0}$, DE (9) has one root which is removed when $u>u_{*}^{1}$. This evolution of the fundamental DR surface mode is shown in Figs. 6 for different values of $\epsilon$ and $\kappa$. These roots are shown in Fig. 8 where the dispersion curves (DCs) $\gamma=\gamma(\kappa)$ decrease posessing thus anomalous dispersion with negative slopes. The emergence and removal of roots $x_{1, d}^{(2)}$ and $\gamma_{1, d}^{(2)}$ take place first on an interval $\nu_{1}^{1}<x<\nu_{1}^{0}$ and then is repeated on every interval $\nu_{n}^{1}<x_{1, d}^{(q)}<\nu_{n}^{0}$.

A further increase of $\kappa$ (and $u$ ) until $u$ reaches $u=\nu_{1}^{1}$ (where $G_{d}(\gamma)$ has singularity) and then exceeds this value leads to the emergence of the DE root associated with the smaller (left) intersection point of $F_{d}(\gamma)$ and $G_{d}(\gamma)$ in Fig. 6; the corresponding DCs with anomalous dispersion are shown in Fig. 9. The DCs with regular dispersion associated with the greater (right) intersection point of $F_{d}(\gamma)$ and $G_{d}(\gamma)$ in Fig. 6 are in Fig. 9; they give rise to higher-order surface waves existing for all $\kappa>0$ and similar to those in DR with positive permittivity.

\subsection{Metamaterial GL}

Determine the conditions of the existence of symmetric surface waves in GL for negative $\epsilon$ resulting from the occurrence of roots of DE (11) on the interval $\Gamma(\epsilon)$ where DE is defined and considered. Note first that $G_{g}(\gamma)$ preserves for any $s>1$ all properties of $G_{d}(\gamma)$ indicated in item (id) in the previous section and we will therefore do not repeat them referring to (id) above. Following the above analysis performed for DR, highlight some properties of the functions $F_{g}=F_{g}(\gamma)$ and $G_{g}=G_{g}(\gamma)$ entering the DE important for our analysis.

(ig) $F_{g}=F_{g}(x)$ is positive on $\Gamma(\epsilon)$ with $F_{g}(\gamma=1)=$ $F_{g}(x=u)=\mathrm{F}_{g}(u)=u \operatorname{Cotj}(u)>0$ for $\kappa, s$ such that $0<u<\min \left(\sqrt{\epsilon}, h_{1}^{0}(s)\right) ; \lim _{x \rightarrow+0} x \operatorname{Cotj}(x)=0$ (in view of the asymptotic expansions of the Bessel and Neumann functions in a vicinitiy of zero), so that one can set $F_{g}(\gamma=$ $\sqrt{|\epsilon|})=0$ for all $\kappa>0, s>1$ and $F_{g}(\kappa=0)=0$. On the other hand, $G_{g}=G_{g}(x)$ is negative on $\Gamma(\epsilon)$ with $G_{g}(\gamma=1)=G_{g}(x=u)=0$ and $G_{g}(\gamma=\sqrt{|\epsilon|})=$ $G_{g}(x=0)=\mathrm{K}(u)<0$ (for negative $\epsilon$ ) Thus $F_{g}(\gamma)$ and $G_{d}(\gamma)$ have different signs on the whole interval $\Gamma(\epsilon)$ for $\kappa, s$ such that $0<u<\min \sqrt{|\epsilon|}, h_{1}^{0}(s)$.

(iig) $F_{g}(\gamma)$ has singularities (poles) on $\Gamma(\epsilon)$ at $\gamma=$ $\gamma_{n, g}(s)=\sqrt{|\epsilon|-\left(\frac{h_{n}^{0}(s)}{\kappa}\right)^{2}}$ corresponding to $x=x_{n, d}=$ $\kappa \sqrt{|\epsilon|-\gamma_{n, g}^{2}}=h_{n}^{0}(s)$ if $\kappa>0$ and $s>1$ are such that $\gamma_{n, g} \in \Gamma(\epsilon)(n=1,2, \ldots)$ for a given $\epsilon<-1$.

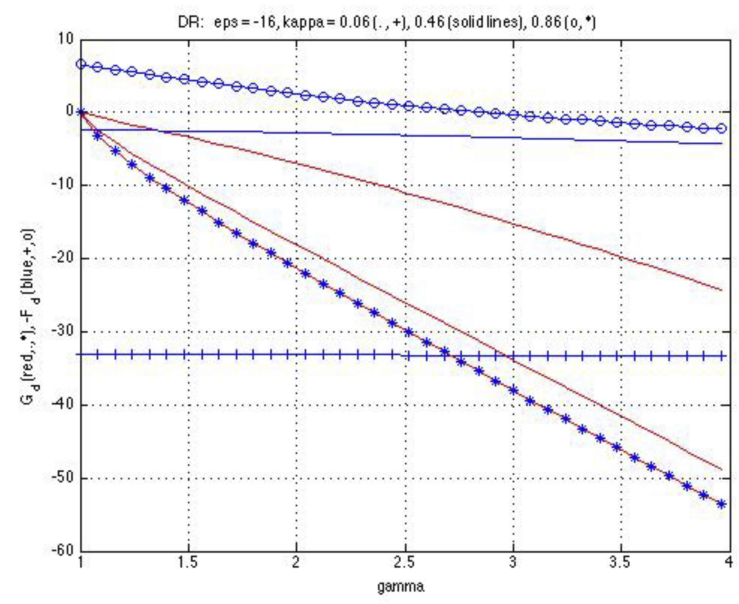

Figure 5: Plots of $F_{d}=F_{d}(\gamma)$ (blue) and $G_{d}=G_{d}(\gamma)$ (red) of DE (9) showing the evolution of the fundamental DR surface mode for $\epsilon=-16$ at $\kappa=0.06$ (upper red curve, +), 0.46 (middle curves), and 0.86 (o, *).

Now we can formulate the conditions governing absence or presence of symmetric surface waves in GL in terms of parameter $u$ and that are largely similar to those proved above for DR:

If $\kappa, s$ are such that, for a given $\epsilon<-1$, we have $0<u<h_{1}^{0}(s)$, GL does not support any symmetric surface waves. As parameter $u$ increases $F_{g}(\gamma)$ acquires singularities $\gamma_{1, g}(s)$ on $\Gamma(\epsilon)$ and DE (11) two roots $\gamma_{1, g}^{(q)}(s)=\sqrt{|\epsilon|-\left(\frac{x_{1, g}^{(q)}(s)}{\kappa}\right)^{2}}$ with $h_{1}^{0}(s)<x_{1, g}^{(q)}(s)<$ $h_{1}^{1}(s), q=1,2$ (similarly to DE (9)) with $x_{1, g}^{(1)}(s)<x_{1, g}^{(2)}(s)$ and $\gamma_{1, g}^{(1)}(s)>\gamma_{1, g}^{(2)}(s)$. The root $x_{1, g}^{(2)}(s)$ is in a left vicinity of $x=u$ and the corresponding $\gamma_{1, g}^{(1)}(s)$ is in a right vicinity of $\gamma=1$. This root is preserved until $F_{g}(x)$ vanishes at $x=h_{1}^{1}(s)$ becoming positive as $u$ increases (together with $\kappa$ ) which leads to the removal of this root. That is, the second greater root $x_{1, g}^{(2)}$ tends to $x=u$ (and $\gamma_{1, g}^{(2)}$ characterized by anomalous dispersion shown in Fig. 7 to $\gamma=1$ ) and then disappears as $u$ increases.

The root $\gamma_{1, g}^{(1)}$ shifts towards the (first) singularity $\gamma_{1, g} \in$ $\Gamma(\epsilon)$ of $F_{g}=F_{g}(\gamma)$ and remains for all $\kappa>0, s>1$. This evolution takes place first on an interval $h_{1}^{0}(s)<x<h_{1}^{1}(s)$ and then is repeated on every interval $h_{n}^{0}(s)<x<h_{n}^{1}(s)$.

Figure 7 plots $F_{g}=F_{g}(\gamma)$ (red, black, green) and $G_{g}=G_{g}(\gamma)$ (blue) entering DE (11), (12) that illustrate evolution (emergence and removal) of the roots $\gamma_{1, g}^{(1,2)}$ for $\epsilon=-4, \kappa=1$ and three different values of $s$. Figure 7 illustrates the anomalous dispersion of $\gamma_{1, g}^{(2)}$ showing how $\gamma_{1}(\kappa)=\gamma_{1, g}^{(2)}(\kappa)$ shifts to $\gamma=1$. Figure 11 displays plots of $\gamma_{1}=\gamma_{1, g}^{(1)}(s)$ solving DE (11), (12) for different $\kappa$ and two different values of $\epsilon$. 


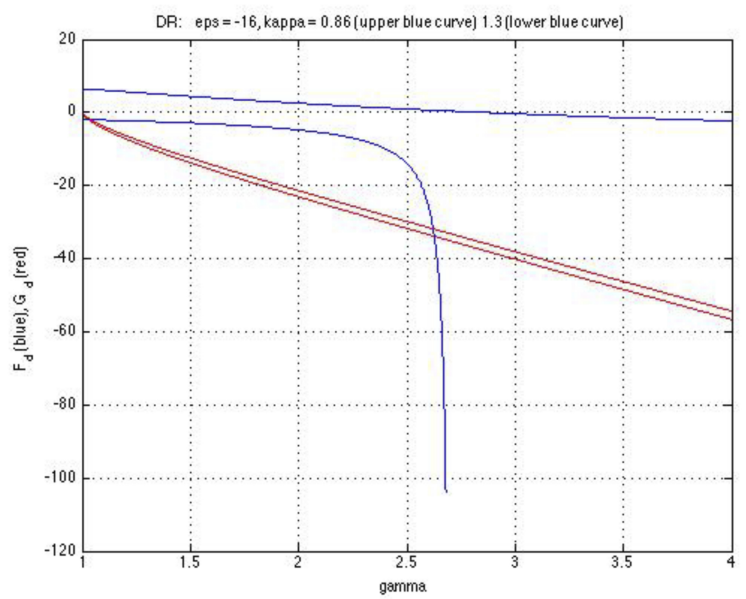

Figure 6: Plots of $F_{d}=F_{d}(\gamma)$ (blue) and $G_{d}=G_{d}(\gamma)$ (red) of DE (9) showing the evolution of the fundamental DR surface mode for $\epsilon=-16$ at $\kappa=0.86$ (upper curves) and 1.3 ( (lower curves).

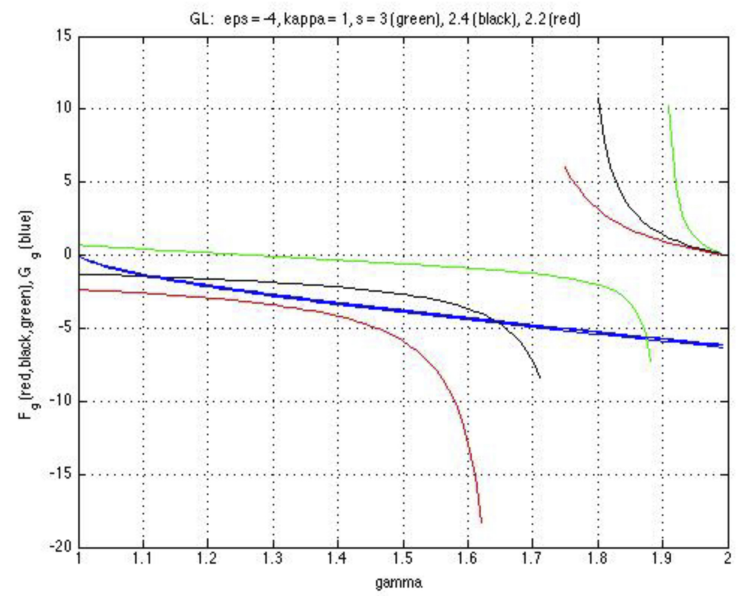

Figure 7: Plots of $F_{g}=F_{g}(\gamma)$ (red, black, green) and $G_{g}=$ $G_{g}(\gamma)$ (blue) in DE (11), (12) showing the evolution of the fundamental GL surface mode for $\epsilon=-4, \kappa=1$ and $s=3$ (green), 2.4 (black) and 2.2 (red).

\section{Properties of symmetric surface waves in metamaterial DR and GL}

Summarize most important properties of symmetric surface waves in metamaterial DR and GL discovered on the basis of the results of this study. These properties differ significantly from those of surface waves in 'standard' DR and GL filled with the media having positive permittivity.

1. Metamaterial medium leads to the phenomen inherent to both DR and GL which is not registered for waveguides filled with 'standard' media: occurrence of two symmetric surface waves emerging in pairs on disjoint frequency intervals. One of these waves (with a smaller relative propagation constant which

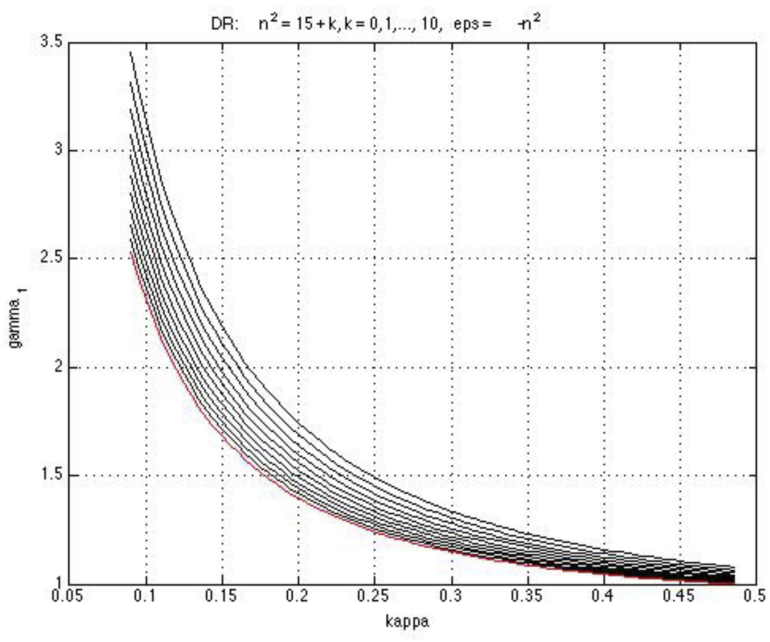

Figure 8: Plots of $\gamma_{1}=\gamma_{1}(\kappa)$ solving DE (9) for the fundamental DR surface modes vs $\kappa$ for $\epsilon=-15-k$ from $k=0$ (upper black curve) to $k=10$ (lower red curve).

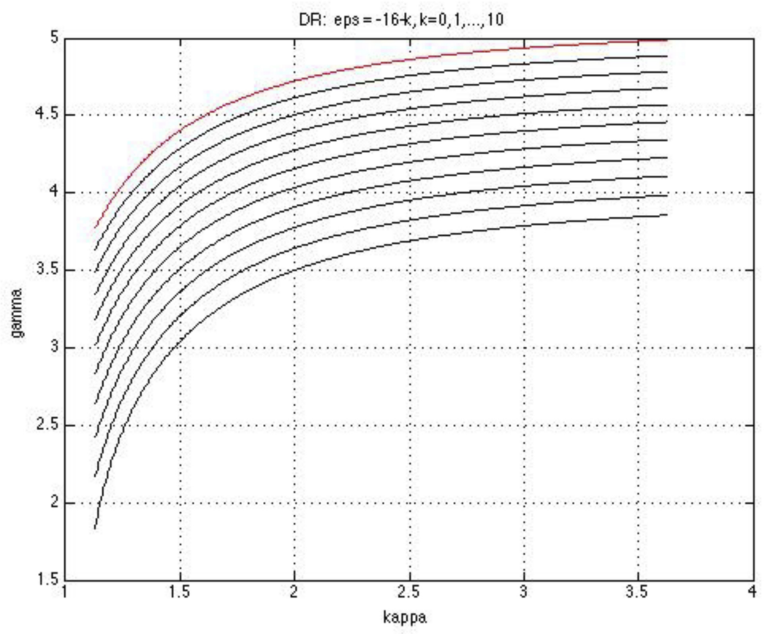

Figure 9: Plots of $\gamma_{2}=\gamma_{2}(\kappa)$ solving DE (9) for a higherorder DR surface modes at $\epsilon=-16-k$ from $k=0$ (lower black curve) to $k=10$ (upper red curve) corresponding to smaller (left) intersection points of $F_{d}(\gamma)$ and $G_{d}(\gamma)$ in Fig. 6.

tends to 1 as relative radius $\kappa$ increases) exists over a relatively short interval and is characterized by anomalous dispersion, and the other does not cease and is preserved for greater values of relative radius $\kappa$ and $s$. The former wave has two cutoff frequencies and the short intervals where it exists are repeated over the whole range $\kappa>\kappa^{*}$. Emergence and removal of waves are governed by the parameter $u=\kappa \sqrt{n^{2}-1}=\kappa \sqrt{|\epsilon|-1}$ which may be called the shifted electric radius of the waveguide.

2. For metamaterial DR, a fundamental symmetric surface wave appears at much smaller $\kappa$ than in the case 
of standard medium, so that the cutoff value for this fundamental mode may be by an order of magnitude less than for standard DR. The fundamental wave in DR has lower and upper cutoffs $\kappa_{l o, 1}^{*}(\epsilon)$ and $\kappa_{u p, 1}^{*}(\epsilon)$ determined from the conditions $u=\nu_{1}^{0}$ and (15).

3. A metamaterial GL does not support the Sommerfeld wave which exists in GL covered by an arbitrarily thin standard dielectric. A fundamental surface wave in metamaterial GL has a rather high lower cutoff value of parameter $s$, the ratio of the external and internal GL radii, determined from the condition $u=h_{1}^{0}(s)$ where $h_{1}^{0}(s)$ is the first zero (w.r.t. $x$ ) of the function $\Phi_{2}$ in (11). Figure 11 displays propagation constants of the fundamental surface wave in metamaterial GL in the range of $s$-values above the cutoff.

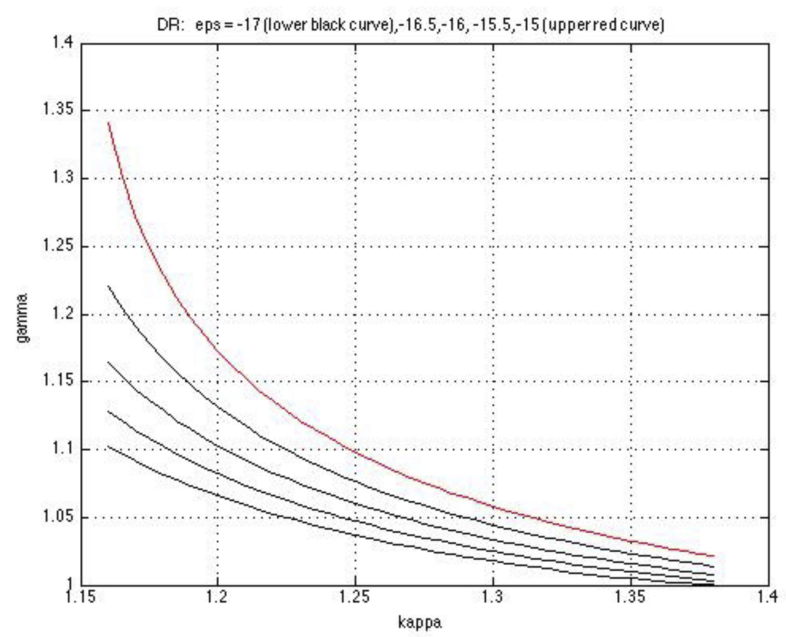

Figure 10: Plots of $\gamma_{2}=\gamma_{2}(\kappa)$ solving DE (9) for a higherorder DR surface modes at $\epsilon=-15-0.5 k$ from $k=0$ (upper red curve) to $k=4$ (lower black curve) corresponding to greater (right) intersection points of $F_{d}(\gamma)$ and $G_{d}(\gamma)$ in Fig. 6.

Exemplify the frequency ranges and cutoff values where e.g. the mentioned fundamental symmetric surface waves in DR exists. Consider a DR of the radius $a=0.4$ [cm] filled with metamaterial having $\epsilon=-5$ and $\mu=-1$ $\left(n^{2}=5\right.$ ) that occurs e.g. at the frequencies around $f=$ $f^{*}=12 G H z$ for a double-ring-resonator metamaterial [3]. In this case $k_{0}=k_{0}^{*}=\frac{2 \pi f^{*}}{c} \approx 2.5, \kappa^{*}=k_{0}^{*} a \approx 1$, and $u^{*}=\kappa^{*} \sqrt{|\epsilon|-1}=2 \kappa^{*} \approx 2$. The lower (when the wave emerges) and upper (when it ceases) calculated cutoffs $\kappa_{l o, 1}^{*}(-5) \approx 0.15$ and $\kappa_{u p, 1}^{*}(-5) \approx 0.55$ so that for the chosen radius the corresponding frequency interval where the fundamental symmetric surface waves exists is from 1.8 to $6.6 \mathrm{GHz}$. For a DR with the same radius filled with standard dielectric having $\epsilon=5$ (and $\mu=1$ ) the lower cutoff $\kappa=\kappa_{l o, 1}^{*}(5)$ at which the fundamental symmetric surface wave starts to exist is determined from the equality $u=2 \kappa_{l o, 1}^{*}(5)=\nu_{1}^{0} \approx 2.4$ which yields $\kappa_{l o, 1}^{*}(5) \approx 1.2$ and $f \approx \kappa_{l o, 1}^{*}(5) \frac{3 \cdot 10^{10}}{0.8 \pi} \approx 1.2 \kappa_{l o, 1}^{*}(5) \cdot 10^{10}=1.44 \cdot 10^{10}=$ $14.4 \mathrm{GHz}$

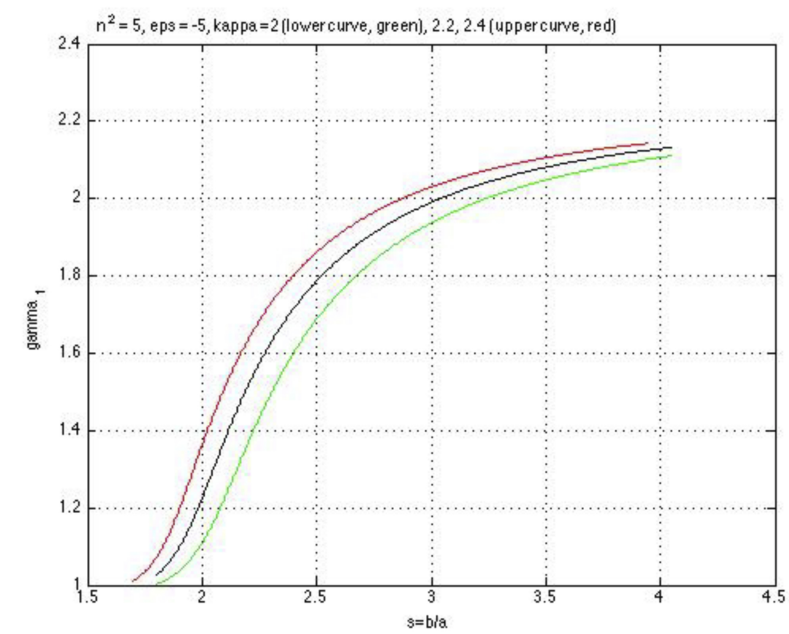

Figure 11: Plots of $\gamma_{1}=\gamma_{1}(\kappa)$ solving DE (11), (12) of the fundamental GL surface modes vs $s=b / a$ for $\kappa=2$ (lower green curves), 2.2, and 2.4 (upper red curves) at $\epsilon=$ -4 .

\section{Conclusion}

We have proved the existence of symmetric surface waves in DR of circular cross section and GL filled with metamaterial media and have determined the location of the corresponding sets of roots of the DEs. A technique for correct mathematical investigation of DEs has been elaborated.

On the basis of in-depth analysis using this technique and numerical solution of DEs we have shown that symmetric surface waves in metamaterial DR and GL differ substabtially from those in 'standard' guides (filled with media having positive permittivity). In particular, symmetric surface waves exist in pairs on disjoint frequency intervals; one of them is characterized by anomalous dispersion, has two cutoff frequencies, and the frequency intervals where it exists are repeated over the infinite range of variation of the relative waveguide radius $\kappa$. For a metamaterial DR, a fundamental symmetric surface wave appears at much smaller $\kappa$ than in DR filled with standard medium and the cutoff value for this fundamental mode may be by an order of magnitude less than for standard DR. Unlike GL covered by an arbitrarily thin standard dielectric, a metamaterial GL does not support the Sommerfeld wave. A fundamental surface wave in metamaterial GL has a high lower cutoff value in terms the ratio $s$ of the external and internal GL radii.

The methods developed in this study and the results obtained will be used as a mathematical background to determine real and complex wave spectra for broader families of open dielectric and metamaterial waveguides including multi-layered fibers. 


\section{Acknowledgement}

The authors acknowledge support of the Swedish Institute,

project Largescale.

\section{References}

[1] A. Sihvola, Metamaterials in electromagnetics, Metamaterials, 1: 3-10, 2007.

[2] D.R. Smith, J.B. Pendry, C.K. Wiltshire, Metamaterials and negative refraction index, Science, 305: 788792, 2004

[3] D.R. Smith, et al, Design and Measurement of Anisotropic Metamaterials that Exhibit Negative Refraction, IEICE Trans. Electron, Vol. E87-C, No. 3, 359-370, 2004.

[4] Y.V. Shestopalov, Resonant States in Waveguide Transmission Problems, Progress In Electromagnetics Research B, Vol. 64, 119-143, 2015.

[5] Y.V. Shestopalov, E.A. Kuzmina, A.B. Samokhin, On a Mathematical Theory of Open Metal-Dielectric Waveguides, Forum for Electromagnetic Research Methods and Application Technologies (FERMAT), 2014.

[6] A.P. Anyutin, I.P. Korshunov, A.D. Shatrov, High-Q Resonances of Surface Waves in Thin Metamaterial Cylinders, J. Commun. Technol. Electron., Vol. 59, No. 11, 1087-1091, 2014.

[7] V. Shestopalov, Y. Shestopalov, Spectral Theory and Excitation of Open Structures, IEE, London, 1996.

[8] Y.V. Shestopalov, E.A. Kuzmina, Waves in a Lossy Goubau Line, Proc. 10th European Conference on Antennas and Propagation EuCAP 2016, 1107-1111, 2016.

[9] E. Kartchevski, et al, Mathematical analysis of the generalized natural modes of an inhomogeneous optical fiber, SIAM Journal on Applied Mathematics, Vol. 65, 2033-2048, 2005. 